\title{
Review Article \\ Osteopetrosis and Its Relevance for the Discovery of New Functions Associated with the Skeleton
}

\author{
Amélie E. Coudert, ${ }^{1}$ Marie-Christine de Vernejoul, ${ }^{2}$ \\ Maurizio Muraca, ${ }^{3}$ and Andrea Del Fattore ${ }^{3}$ \\ ${ }^{1}$ Institut National de la Santé et de la Recherche Médicale U1138, Centre de Recherche des Cordeliers, Paris, France \\ ${ }^{2}$ Institut National de la Santé et de la Recherche Médicale U1132, Hôpital Lariboisière, Paris, France \\ ${ }^{3}$ Regenerative Medicine Unit, Bambino Gesù Children's Hospital, IRCCS, Piazza Sant'Onofrio 4, 00165 Rome, Italy
}

Correspondence should be addressed to Andrea Del Fattore; andrea.delfattore@opbg.net

Received 6 August 2014; Revised 16 October 2014; Accepted 30 October 2014

Academic Editor: Cristina Sobacchi

Copyright ( 2015 Amélie E. Coudert et al. This is an open access article distributed under the Creative Commons Attribution License, which permits unrestricted use, distribution, and reproduction in any medium, provided the original work is properly cited.

\begin{abstract}
Osteopetrosis is a rare genetic disorder characterized by an increase of bone mass due to defective osteoclast function. Patients typically displayed spontaneous fractures, anemia, and in the most severe forms hepatosplenomegaly and compression of cranial facial nerves leading to deafness and blindness. Osteopetrosis comprises a heterogeneous group of diseases as several forms are known with different models of inheritance and severity from asymptomatic to lethal. This review summarizes the genetic and clinical features of osteopetrosis, emphasizing how recent studies of this disease have contributed to understanding the central role of the skeleton in the whole body physiology. In particular, the interplay of bone with the stomach, insulin metabolism, male fertility, the immune system, bone marrow, and fat is described.
\end{abstract}

\section{Introduction}

Bone is a dynamic tissue which undergoes continuous selfrenewal, and bone homeostasis relies on functional equilibrium among three types of cells: osteoclasts essential for bone resorption, osteoblasts responsible for bone matrix formation, and osteocytes involved in the reception and transduction of mechanical stimuli and in the regulation of osteoclast/osteoblast differentiation and function [1]. The balance between bone synthesis and resorption is finely tuned and any perturbations of this balance in adults trigger bone disease. Human osteopetrosis was first described by AlbersSchönberg in 1904 [2]. Osteopetrosis (osteo: bone and petros: stone) regroups a set of rare, heterogeneous, and inherited bone diseases characterized by increased bone mass. Osteopetrosis is therefore an osteocondensing disease. In principle, two causes could give rise to this osteocondensing phenotype: increased bone formation or failure of resorption by osteoclasts. However, osteopetrosis is known to result from defective osteoclast differentiation or function $[3,4]$.
Important progress has been made during the past decades in understanding the molecular mechanisms underlying the development of hereditary diseases characterized by increased bone mass $[3,5]$.

Our objective in this review is not to give a detailed description of all the sclerosing bone diseases; such information can be found in other reviews $[3,4,6,7]$. Instead, we discuss recent findings regarding osteopetrosis and how the study of this disease has contributed to new understanding of functions associated with the skeleton [8-10].

\section{Osteoclasts}

Osteoclasts are highly specialized cells responsible for the dissolution of bone mineral and for the degradation of organic matrix. This activity is essential to bone remodeling and mineral homeostasis [8].

Osteoclasts are multinucleated cells (containing up to 50 nuclei), derived from the fusion of mononuclear cells belonging to the monocyte-macrophage lineage. Under the 
TABLE 1: Genes mutated in osteopetrotic patients.

\begin{tabular}{lccll}
\hline $\begin{array}{l}\text { Osteopetrosis } \\
\text { form }\end{array}$ & Genetic transmission & Gene & Mutation type & Protein \\
\hline & TCIRG1 & Loss of function & $\alpha 3$ subunit V-ATPase \\
& CLCN7 & Loss of function & Chloride channel 7 \\
& OSTM1 & Loss of function & Osteopetrosis associated transmembrane protein \\
ARO & Autosomal recessive & PLEKHM1 & Loss of function & Pleckstrin homology domain containing family M, member I \\
& & SNX10 & Loss of function & Sorting nexin 10 \\
& TNFSF11 & Loss of function & Receptor activator for nuclear factor $\kappa$ B ligand \\
& & TNFRSF11A & Loss of function & Receptor activator for nuclear factor $\kappa \mathrm{B}$ \\
\hline IRO & Autosomal recessive & CAII & Loss of function & Carbonic anhydrase II \\
\hline ADO II & Autosomal dominant & CLCN7 & Dominant negative & Chloride channel 7 \\
\hline
\end{tabular}

influence of factors secreted by osteoblasts and/or stromal cells present in the bone microenvironment, these precursors differentiate into osteoclasts [1].

The osteoclast differentiation pathway and the molecules involved are now well established. M-CSF (macrophage colony stimulating factor) is expressed by osteoblasts and binds the $\mathrm{c}$-fms receptor on osteoclast precursors, stimulating their proliferation and the expression of RANK (receptor activator of NF- $\kappa \mathrm{B}$ ) receptor. The interaction of RANK-L, expressed and secreted by osteoblasts and stromal cells, with its receptor propels the fusion of osteoclast progenitors to form a giant multinucleated cell. Osteoprotegerin inhibits osteoclast differentiation by acting as a receptor decoy for RANK-L [1].

A terminally differentiated osteoclast is able to degrade extracellular bone matrix by the action of specific proteins. To resorb bone matrix, osteoclasts must be perfectly polarized with a ruffle border and a sealing zone. These two features allow the creation of a resorption lacuna into which $\mathrm{H}^{+}$ ions are actively secreted in order to acidify it, leading to dissolution of bone matrix hydroxyapatite [1]. Creation of the acidic compartment requires a continuous source of protons. Type II carbonic anhydrase (CAII) hydrates $\mathrm{CO}_{2}$ to form carbonic acid, which spontaneously dissociates into protons and $\mathrm{HCO}_{3}{ }^{-}$ions. The protons are actively transported into the resorption lacuna by a vacuolar ATPase proton pump located in the ruffled border domain $[1,11]$. The $\mathrm{HCO}_{3}{ }^{-}$ion is exchanged with $\mathrm{Cl}^{-}$by a bicarbonate/chloride antiport on the basolateral membrane of the cell. The chloride ion is translocated into the resorption lacuna through chloride channel 7 (ClCn7), recently reclassified as chloride/proton antiport. The acidic environment promotes the dissolution of inorganic content and also exposes the organic matrix, which is then ready to be digested by secreted proteolytic enzymes $[1,11]$.

The collagenous bone matrix is dissolved by two groups of enzymes, the matrix metalloproteases and the lysosomal cathepsins. Cathepsin K especially has been identified as a key enzyme in osteoclast function. This enzyme is secreted into the resorption lacuna and degrades type I collagen in the acidic environment [12].
The acquisition and maintenance of osteoclast membrane polarity require a complex system of vesicle trafficking and ongoing cytoskeletal renewal [1]. One of the proteins involved in these processes is Plekhml (pleckstrin homolog domain containing family $M$ with run domain member 1). This protein plays a crucial role in acidification and trafficking of intracellular vesicles $[13,14]$. A recently discovered protein important for osteoclast trafficking activity is Snx10 (sorting nexin 10). Snx10 belongs to a family of about 30 proteins sharing the PX (phox homology) phospholipid binding domain and is involved in protein trafficking and osteoclast differentiation/function $[15,16]$.

\section{Osteopetrosis}

Osteopetrosis is a generic name for a group of rare genetic bone diseases characterized by osteoclast failure [6]. Several forms are known with different models of inheritance and severity. The adult autosomal dominant type II form or Albert-Schönberg disease classified as mild is sometimes associated with bone symptoms. This is the most frequent form of osteopetrosis observed by rheumatologists. In contrast, the infantile recessive osteopetroses are severe forms and usually lethal in childhood without treatment $[3,5-7,17]$. Mutations in at least 8 genes (Table 1) have been identified as being responsible for osteopetrosis pathogenesis in humans.

3.1. Autosomal Recessive Osteopetrosis. Autosomal recessive osteopetrosis is a severe disease diagnosed in the first months of life owing to a variety of problems [3]. Patients are treated in pediatrics or hematology departments. Sick children have recurrent infections. They also show bruising and frequent bleeding secondary to medullar hyperplasia caused by bony invasion of the medullar space. Cranial nerve compressions can occur leading to blindness and deafness. Neurological defects may also be observed in some patients independently of nerve compressions. X-ray analysis reveals dense bones which are characterized by extreme brittleness. Untreated children usually die during their decade from hemorrhage, pneumonia, anemia, or infection. Hematopoietic stem cell 


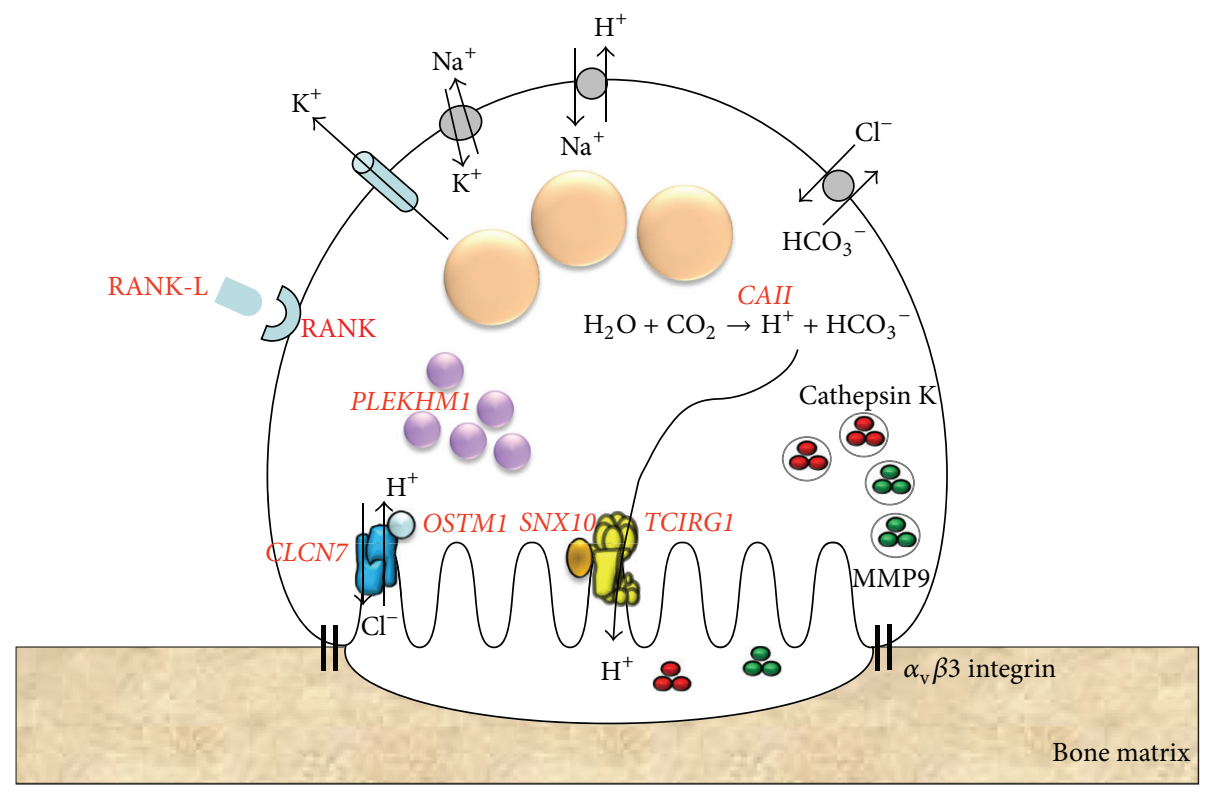

FIGURE 1: Schematic diagram showing an osteoclast and the involved genes in osteopetrosis. Cellular localization and protein involved in osteoclast differentiation and function. The genes mutated in human osteopetrosis are red in bold.

transplantation (HSCT) is the only treatment option currently far available $[3,5,18]$.

Several biological abnormalities can cause this pathology. Generally in ARO, the number of osteoclasts is normal or high, but their acidifying activity, compulsory for bone resorption, is impaired [17]. Several genes are known to be involved in this form of osteopetrosis (Table 1, Figure 1). About $50 \%$ of ARO patients harbors loss-of-function mutations of TCIRG1 which codes for the proton pump VATPase $\alpha 3$ subunit $[17,19]$. Loss-of-function mutations of the CLCN7 gene, coding for chloride channel 7, have also been described in $\sim 10 \%$ of ARO patients [20]. Mutations in OSTM1 (osteopetrosis associated transmembrane protein 1), coding for a protein involved in transport of $\mathrm{ClCn} 7$ to the ruffled border (and considered as a $\beta$ subunit of $\mathrm{ClCn} 7$ ), have been described as causing severe osteopetrosis in $~ 5 \%$ of patients [21-23]. Primary neurological defects can also be present in patients bearing OSTM1 or CLCN7 mutations [20, 23].

Two cases of intermediate forms of ARO caused by PLEKHM1 mutations have been described. An "Erlenmeyer flask" deformity of the distal femora, bone pain, and chondrolysis of the left hip were described in one patient. Interestingly, a brother with the same mutation showed no clinical signs [14]. Recently, a mutation in the SNX10 gene was found in 15 families in which the patients displayed a heterogeneous phenotype. Mild growth retardation, hypocalcemia, hydrocephalus, severe hematological abnormalities, and visual impairment have been described in patients with loss of function mutations of $S N X 10[3,15,16,24]$.

Less than $4 \%$ of ARO patients harbors loss-of-function mutations of TNFSF11, encoding RANK-L, or of TNFRSF11A, encoding RANK receptor, and constitute a distinct subgroup of recessive osteopetrosis. Indeed, bone biopsies from these patients revealed a complete lack of osteoclasts [25-27].
In addition, patients with TNFSF11 mutations exhibit some immune abnormalities and not palpable lymph nodes, but B and T lymphocyte numbers are normal. By contrast, most of the patients with TNFRSF11A mutations have a more severe immunological phenotype with a defect in memory B lymphocyte differentiation and a reduction in immunoglobulins levels [25-27].

Treatment of most recessive forms of osteopetrosis includes HSCT, which restores osteoclast function. However, osteopetrosis caused by TNFSF11 mutations cannot be treated by HSCT, because an osteoblast defect is the basis of this pathology [28]. In practice, a molecular genetic diagnosis should be made before transplantation to ensure that the pathology is not due to a RANK-L mutation.

3.2. A Specific Intermediate Recessive Osteopetrosis (IRO): Type II Carbonic Anhydrase Deficiency. In 1983, an autosomal recessive osteopetrosis syndrome associated with renal tubular acidosis was described [29]. The clinical signs of the affected patients are highly variable. Mental deficiency is frequent, but not always present. Optical nerve compression and dental malocclusions can occur. Renal tubular acidosis can explain the hypotonia, apathy, and muscular weakness occurrence in some patients. By radiography CAII deficiency resembles other forms of osteopetrosis, but brain calcifications can develop during childhood and osteosclerosis and bone modeling spontaneously decrease instead of increasing in the course of pathology evolution. Metabolic acidosis occurs during the neonatal period, and renal tubular acidosis, both proximal and distal, has been described $[29,30]$.

CAII is expressed in many different tissues including brain, kidney, red blood cells, cartilage, lung, and digestive mucosa. All patients with this pathology have a selective defect involving CAII expressed in erythrocytes [31]. 
3.3. Type II Autosomal Dominant Osteopetrosis (ADOII Also Known as Albert-Schönberg Disease). ADO II is commonly called benign osteopetrosis but presents with an extremely heterogeneous course from asymptomatic to rarely fatal. Prevalence of the pathology has been estimated at 5 per 100 000 [32].

ADOII clinical and radiological signs occur quite late in childhood or in the teens, although earlier occurring has sometimes been reported. ADOII patients usually displayed osteosclerosis at the vertebral level (so-called sandwich vertebrae) and also a bone in bone aspect observed mainly in the iliac bones, but sometimes in other epiphyses. An increase in cranial bone density can also occur. In addition, on radiography, alternating dense and light bands are often seen in iliac bones and at the extremities of long bones [7,33].

The main ADOII complications involve the skeleton [34]. Bone fractures occur in $80 \%$ of patients, with a mean of 3 fractures per patient. A few patients have had more than 10 fractures. The femur is the most fractured bone in this pathology, but fractures can occur on any long bones and even at the posterior arch of the vertebrae, which often leads to a spondylolisthesis. Scoliosis is not rare. Hip arthritis is frequent (in $50 \%$ of the cases) and could be due to excessive stiffness of the subchondral bone. Arthritis can occur in other locations as well. Mandibular osteomyelitis is often associated with dental abscess or carious cavity. Cranial nerve compressions caused by osteosclerosis are rare. Auditory or visual impairment occurs in less than $5 \%$ of affected individuals $[7,33]$.

Orthopedic treatment is often necessary to treat fractures and arthritis. Arthropathies are technically difficult and postsurgical complications, such as strengthening delay, infections, and pseudoarthritis are frequent (50\% of cases) due to bone stiffness. The penetrance of ADOII is $60-90 \%$. Disease severity is highly variable, even within the same family [33]. For example, in 3 families in which most of the affected individuals expressed only a mild form of ADOII, some members exhibited anemia and blindness caused by optical nerve compression. This phenotype has been called intermediate osteopetrosis because of its overlap with that of mild ARO [33].

About $70 \%$ of patients affected by ADOII harbors heterozygous dominant negative mutations of the CLCN7 gene (Figure 1, Table 1) [33]. In the remaining $~ 30 \%$ of cases, no mutations in CLCN7 gene sequences were found, suggesting involvement of further genes in the pathogenesis of this form of osteopetrosis [33].

\section{The Relevance of Osteopetrosis Studies to New Understanding of Functions Associated with the Skeleton}

4.1. Osteopetrosis and the Bone-Stomach Interaction. Osteopetrorickets is a bone disorder characterized by increase of bone mass with a defect of skeletal mineralization. Schinke and coauthors performed histological analysis of undecalcified bone biopsies of 21 patients who received a diagnosis of osteopetrosis. In patients with loss-of-function mutations in the TCIRG1 gene, an increase of unmineralized bone matrix osteoid was observed. The same pathological enrichment of osteoid was confirmed in oc/oc mice carrying a loss-offunction mutation of the tcirg1 gene, while no increase was revealed in osteopetrotic $\mathrm{scr}^{-1-}$ mice [35].

The increase of osteoid volume was associated with hypocalcemia, due to a defect of intestinal calcium uptake. Indeed it was shown that TCIRG1 is also expressed in the fundus, a region of the stomach involved in gastric acidification, and loss-of-function mutations induce hypochlorhydria and reduced intestinal calcium uptake in both humans and mice [35].

This study was fundamental in demonstrating a physiological link between the stomach and bone. Gastric acidification is a prerequisite for efficient intestinal calcium uptake; in hypochlorhydria, intestinal calcium uptake is lowered leading to parathyroin hormone (PTH)-dependent activation of osteoclasts and an osteoporosis phenotype. In the case of lossof-function mutation of TCIRG1, intestinal calcium uptake is reduced and PTH-dependent stimulation of bone resorption is blocked, resulting in an osteopetrorickets phenotype [35].

Barvencik et al. performed histomorphometric analysis of bone biopsies of 9 osteopetrotic patients with loss-ofmutation in the TCIRG1, CLCN7, and TNFSF11A genes [36]. Pathological enrichment of nonmineralized bone matrix was observed in all cases with TCIRG1 mutations. In contrast, there was no sign of osteopetrorickets in patients with CLCN7 and TNFSF11A gene mutations [35-37].

4.2. Osteopetrosis and Insulin Metabolism. Osteopetrosis studies were fundamental to understand the link between bone and osteocalcin signaling. Osteocalcin is a small protein embedded in bone matrix. Osteocalcin can exist in two different forms, undercarboxylated and carboxylated on 3 glutamic acid residues $[10,38]$. The carboxylated form has high affinity for the hydroxyapatite, facilitating its engraftment in the bone matrix. It was shown that acidic $\mathrm{pH}$ can decarboxylate proteins [39]. Ferron et al. investigated whether acidic bone resorption lacuna promotes the decarboxylation of osteocalcin. Indeed they observed that in $o c / o c$ mice the levels of undercarboxylated osteocalcin were reduced by $30 \%$ compared to wild-type animals. Similar features were observed in wild-type mice that received fetal liver hematopoietic stem cells (HSCs) from oc/oc mice confirming the relevance of osteoclast function in osteocalcin-insulin signaling. Moreover they observed that $o c / o c$ mice were glucose intolerant, with reduced serum insulin levels, pancreas insulin content, and insulinexpression in the pancreas [40,41].

Interestingly, it was shown that osteopetrotic patients affected by autosomal dominant osteopetrosis with osteoclast acidification defects have lower levels of insulin and a lower undercarboxylated/carboxylated osteocalcin ratio but diabetes was not reported [40, 41].

4.3. Osteopetrosis and Male Fertility. Osteocalcin is very important for the cross talk between bone and the systems responsible for male fertility [42, 43]. Karsenty's group showed that osteocalcin is able to stimulate, in a cAMP 
response element binding (CREB) protein-dependent manner, the production of testosterone by testes. This function is mediated by the interaction of osteocalcin with GPRC6A, a G-coupled receptor expressed in Leydig cells [42, 43].

In 1997 Cohen et al. [44] showed that op/op mice (which lack colony stimulating factor $1, \mathrm{CSF}-1$ ) have reduced mating ability, low sperm numbers, and low serum testosterone levels due to decreased Leydig cell steroidogenesis. The study also showed how CSF-1 is essential for the development and function of the hypothalamic-pituitary-gonadal axis. Further studies in osteopetrotic animal models will be important to confirm the interaction between bone and male fertility.

4.4. Osteopetrosis and the Immune System. It now well established that there is a tight correlation between bone and the immune system, which has led to a new discipline called osteoimmunology. This research area is just now expanding and we are beginning to better understand the relevance of this interplay in bone diseases [45].

Many osteopetrotic animals are characterized by immunological defects. Associated defects in B cell function were attributed to mutations in genes involved in osteoclast differentiation or function or to an abnormal medullary microenvironment. oc/oc mice display a block at the pro-B to pre-B cell transition, which is due to a defect of the bone microenvironment rather than to a cell autonomous defect of B cells, because in vitro experiments showed that B cell progenitors isolated from osteopetrotic mice were able to differentiate into immature B cells [46].

Moreover it was shown that rankl-/- mice display an immunological defects. Apart the alterations of B cell differentiation, Kong and coauthors described a reduction in thymus size and a block of thymocyte development at the $\mathrm{CD} 4^{-} \mathrm{CD} 8^{-} \mathrm{CD} 44^{-} \mathrm{CD} 25^{+}$stage [47]. These effects are correlated with many functions exerted by RANKL in the immune system [48].

Many studies have been published regarding the effects on osteoclast differentiation and function following alterations of immune cells [45]. The involvement of T regulatory cells (Treg) is still under investigation. In particular, it was shown that animal overexpressing the transcription factor FoxP3 (forkhead box P3) displayed osteopetrotic phenotype with increased bone mass and reduced osteoclast number and activity [49]. Moreover in vitro experiments suggested that Treg cells could inhibit osteoclast differentiation and function by suppression of cytoskeletal reorganization [50].

4.5. Osteopetrosis and Bone Marrow. Bone and bone marrow can be considered as two distinct compartments of the same functional unit, the bone-bone marrow organ. Perturbations to one of the compartments typically affect the other as well [51].

Indeed it was shown that dysfunction of osteoclast activity results in aberrant formation of the HSC niche, leading to retention of HSC in the spleen. The frequency and absolute number of LinnegScal ${ }^{+} \mathrm{CKit}^{+}$(LSK cells) were decreased by $90 \%$ and $99.8 \%$, respectively, in the bone marrow of $o c / o c$ mice compared to controls. This alteration was associated with a defect of mesenchymal stem cells to differentiate into osteoblasts. The effect was revealed by a dramatic reduction in the expression of the osteoblast markers Runx2, Alp, Osteocalcin and Bsp and a reduced proportion of cells expressing CD51 and the integrin $\alpha 5$ (CD49e). The study showed that osteoclasts promote the formation of the HSC niche, regulating the osteoblast differentiation important for the niche [52]. Indeed the authors showed that the absence of osteoclast activity affects formation of the bone marrow HSC niche and impairs ability of mesenchymal stem/stromal cells to recruit hematopoietic progenitor cells. Moreover the restoration of osteoclast function by treatment with $\mathrm{CD} 45^{+} \mathrm{Scal}^{+}$cells reestablishes normal levels of hematopoietic progenitors in the bone marrow $[46,53]$.

4.6. Osteopetrosis and Fat. The relationship between bone and adipose tissue is an area of intensive investigations because molecules involved in bone-fat interactions could be used as pharmacological targets to prevent osteoporosis and bone fractures [54]. In particular, the involvement of peroxisome proliferator-activated receptor- $\gamma$ (PPAR- $\gamma$ ) was studied. PPAR- $\gamma$ is a nuclear receptor and acts as a heterodimer with retinoid X receptor. Ligands for PPAR- $\gamma$ include long-chain fatty acid and synthetic compounds such as thiazolidinedione [55]. PPAR- $\gamma$ functions are associated with activation of the adipogenesis and inhibition of the osteoblastogenesis $[56,57]$.

Moreover Wan et al. investigated PPAR- $\gamma$ function in osteoclasts [58]. The authors used TieCre/flox mice to delete PPAR- $\gamma$ in osteoclasts. These mice developed increased bone mass with a parallel reduction of bone marrow cavities and extramedullary hematopoiesis. Indeed deletion of PPAR- $\gamma$ resulted in impaired osteoclast differentiation and activity, since it regulates $\mathrm{c}$-fos expression involved in RANKL signaling [58].

Moreover Cock et al. demonstrated that the absence of PPAR- $\gamma$ in white adipose tissue led to lipodystrophy, increased bone mineral density, and extramedullary hematopoiesis in spleen [59]. This interplay between bone and adipose tissue has clinical important implications, since a long-term treatment with the PPAR- $\gamma$ agonist rosiglitazone in patients affected by type 2 diabetes could result in osteoporosis and bone fractures $[54,58]$.

\section{Conclusion}

Rare hereditary diseases inducing a bone condensation have shed new light on several aspects of bone cellular biology that were not well known. Indeed the study of these diseases allowed the identification of new mechanisms of osteoclast differentiation and function and the discovery of new functions associated with the skeleton. Much evidence suggests that the skeleton has a central role in bone physiology since bone disorders usually impact other organs $[8,9,42,43,45$, $53,54,60]$. Osteopetrosis studies were essential to demonstrate these interactions. However, there are some features of these diseases that require further investigation. For example, as in other monogenic diseases, the genotype-phenotype correlation is not always clear and consistent. Indeed, the 
same mutations can give rise to different phenotypes, as exemplified by the CLCN7 gene heterozygous mutations. Moreover, the mutations identified to date explain only $70 \%$ of osteopetrosis cases. Efforts to identify the mutations responsible for the remaining 30\% are on-going [33].

From a pathophysiological point of view, it is worth noting that the pathologies caused by reduced osteoclastic activity such as osteopetrosis lead to frequent fractures. This might be linked to a skeleton elasticity defect, but also to an inability to repair micro damage in bones because of a lower rate of bone turnover. This situation illustrates the well-known discrepancy between the bone quantity and its resistance to mechanical stress. In contrast, pathologies caused by an increase in bone formation due to increased activity of the Wnt signaling pathway (striated osteopathy) or to TGF $\beta$ activating mutations (Camurati Engelmann disease) are not associated with an increased incidence of fractures [7].

In conclusion, further study of osteopetrosis will allow us to better understand the physiology of bone and its impact on the whole body. Moreover our challenge for the future will be to identify new therapeutic approaches for this disabling disease, particularly for those forms for which only palliative intervention is currently available.

\section{Conflict of Interests}

The authors declare that there is no conflict of interests regarding the publication of this paper.

\section{References}

[1] A. del Fattore, A. Teti, and N. Rucci, "Bone cells and the mechanisms of bone remodelling," Frontiers in Bioscience, vol. 4, no. 6, pp. 2302-2321, 2012.

[2] H. E. Albers-Schönberg, "Röntgenbilder einer seltenen Knockenerkrankung," Münchener Medizinische Wochenschrift, vol. 5, pp. 365-368, 1904.

[3] C. Sobacchi, A. Schulz, F. P. Coxon, A. Villa, and M. H. Helfrich, "Osteopetrosis: genetics, treatment and new insights into osteoclast function," Nature Reviews Endocrinology, vol. 9, no. 9, pp. 522-536, 2013.

[4] Z. Stark and R. Savarirayan, "Osteopetrosis," Orphanet Journal of Rare Diseases, vol. 4, no. 1, article 5, 2009.

[5] M. H. Helfrich, "Osteoclast diseases," Microscopy Research and Technique, vol. 61, no. 6, pp. 514-532, 2003.

[6] M. C. de Vernejoul and O. Bénichou, "Human osteopetrosis and other sclerosing disorders: recent genetic developments," Calcified Tissue International, vol. 69, no. 1, pp. 1-6, 2001.

[7] M.-C. de Vernejoul and U. Kornak, "Heritable sclerosing bone disorders: presentation and new molecular mechanisms," Annals of the New York Academy of Sciences, vol. 1192, pp. 269277, 2010.

[8] J. F. Charles and A. O. Aliprantis, "Osteoclasts: more than 'bone eaters,' Trends in Molecular Medicine, vol. 20, no. 8, pp. 449-459, 2014.

[9] A. R. Guntur and C. J. Rosen, "Bone as an endocrine organ," Endocrine Practice, vol. 18, no. 5, pp. 758-762, 2012.

[10] D. J. DiGirolamo, T. L. Clemens, and S. Kousteni, "The skeleton as an endocrine organ," Nature Reviews Rheumatology, vol. 8, no. 11, pp. 674-683, 2012.
[11] K. Henriksen, J. Bollerslev, V. Everts, and M. A. Karsdal, "Osteoclast activity and subtypes as a function of physiology and pathology-implications for future treatments of osteoporosis," Endocrine Reviews, vol. 32, no. 1, pp. 31-63, 2011.

[12] V. Everts, W. Korper, K. A. Hoeben et al., "Osteoclastic bone degradation and the role of different cysteine proteinases and matrix metalloproteinases: differences between calvaria and long bone," Journal of Bone and Mineral Research, vol. 21, no. 9, pp. 1399-1408, 2006.

[13] A. del Fattore, L. van Wesenbeeck, F. de Freitas et al., "A new heterozygous mutation (R714C) of the osteopetrosis gene, pleckstrin homolog domain containing family $\mathrm{M}$ (with run domain) member 1 (PLEKHM1), impairs vesicular acidification and increases TRACP secretion in osteoclasts," Journal of Bone and Mineral Research, vol. 23, no. 3, pp. 380-391, 2008.

[14] L. van Wesenbeeck, P. R. Odgren, F. P. Coxon et al., "Involvement of PLEKHM1 in osteoclastic vesicular transport and osteopetrosis in incisors absent rats and humans," The Journal of Clinical Investigation, vol. 117, no. 4, pp. 919-930, 2007.

[15] M. Aker, A. Rouvinski, S. Hashavia et al., "An SNX10 mutation causes malignant osteopetrosis of infancy," Journal of Medical Genetics, vol. 49, no. 4, pp. 221-226, 2012.

[16] A. Mégarbané, A. Pangrazio, A. Villa et al., "Homozygous stop mutation in the SNX10 gene in a consanguineous Iraqi boy with osteopetrosis and corpus callosum hypoplasia," European Journal of Medical Genetics, vol. 56, no. 1, pp. 32-35, 2013.

[17] C. Sobacchi, A. Frattini, P. Orchard et al., "The mutational spectrum of human malignant autosomal recessive osteopetrosis," Human Molecular Genetics, vol. 10, no. 17, pp. 1767-1773, 2001.

[18] M.-C. de Vernejoul, "Sclerosing bone disorders," Best Practice and Research: Clinical Rheumatology, vol. 22, no. 1, pp. 71-83, 2008.

[19] A. Frattini, P. J. Orchard, C. Sobacchi et al., "Defects in TCIRG1 subunit of the vacuolar proton pump are responsible for a subset of human autosomal recessive osteopetrosis," Nature Genetics, vol. 25, no. 3, pp. 343-346, 2000.

[20] U. Kornak, D. Kasper, M. R. Bösl et al., "Loss of the CIC-7 chloride channel leads to osteopetrosis in mice and man," Cell, vol. 104, no. 2, pp. 205-215, 2001.

[21] N. Chalhoub, N. Benachenhou, V. Rajapurohitam et al., "Greylethal mutation induces severe malignant autosomal recessive osteopetrosis in mouse and human," Nature Medicine, vol. 9, no. 4, pp. 399-406, 2003.

[22] P. F. Lange, L. Wartosch, T. J. Jentsch, and J. C. Fuhrmann, "ClC7 requires Ostml as a $\beta$-subunit to support bone resorption and lysosomal function," Nature, vol. 440, no. 7081, pp. 220-223, 2006.

[23] A. Pangrazio, P. L. Poliani, A. Megarbane et al., "Mutations in OSTM1 (grey lethal) define a particularly severe form of autosomal recessive osteopetrosis with neural involvement," Journal of Bone and Mineral Research, vol. 21, no. 7, pp. 10981105, 2006.

[24] A. Pangrazio, A. Fasth, A. Sbardellati et al., "SNX10 mutations define a subgroup of human autosomal recessive osteopetrosis with variable clinical severity," Journal of Bone and Mineral Research, vol. 28, no. 5, pp. 1041-1049, 2013.

[25] A. Pangrazio, B. Cassani, M. M. Guerrini et al., "RANKdependent autosomal recessive osteopetrosis: characterization of five new cases with novel mutations," Journal of Bone and Mineral Research, vol. 27, no. 2, pp. 342-351, 2012. 
[26] C. Sobacchi, A. Frattini, M. M. Guerrini et al., "Osteoclast-poor human osteopetrosis due to mutations in the gene encoding RANKL," Nature Genetics, vol. 39, no. 8, pp. 960-962, 2007.

[27] A. Villa, M. M. Guerrini, B. Cassani, A. Pangrazio, and C. Sobacchi, "Infantile malignant, autosomal recessive osteopetrosis: the rich and the poor," Calcified Tissue International, vol. 84, no. 1, pp. 1-12, 2009.

[28] N. Lo Iacono, H. C. Blair, P. L. Poliani et al., "Osteopetrosis rescue upon RANKL administration to $\mathrm{Rankl}^{-/-}$mice: a new therapy for human RANKL-dependent ARO," Journal of Bone and Mineral Research, vol. 27, no. 12, pp. 2501-2510, 2012.

[29] W. S. Sly, D. Hewett-Emmett, M. P. Whyte, Y. S. Yu, and R. E. Tashian, "Carbonic anhydrase II deficiency identified as the primary defect in the autosomal recessive syndrome of osteopetrosis with renal tubular acidosis and cerebral calcification," Proceedings of the National Academy of Sciences of the United States of America, vol. 80, no. 9, pp. 2752-2756, 1983.

[30] W. S. Sly, M. P. Whyte, V. Sundaram et al., "Carbonic anhydrase II deficiency in 12 families with the autosomal recessive syndrome of osteopetrosis with renal tubular acidosis and cerebral calcification," The New England Journal of Medicine, vol. 313, no. 3, pp. 139-145, 1985.

[31] R. L. Jilka, J. I. Rogers, R. G. Khalifah, and H. K. Vaananen, "Carbonic anhydrase isozymes of osteoclasts and erythrocytes of osteopetrotic microphthalmic mice," Bone, vol. 6, no. 6, pp. 445-449, 1985.

[32] J. Bollerslev, K. Henriksen, M. F. Nielsen, K. Brixen, and W. Van Hul, "Autosomal dominant osteopetrosis revisited: lessons from recent studies," European Journal of Endocrinology, vol. 169, no. 2, pp. R39-R57, 2013.

[33] M. C. de Vernejoul, A. Schulz, and U. Kornak, "CLCN7-related osteopetrosis," in GeneReviews, 2007.

[34] O. D. Bénichou, J. D. Laredo, and M. C. de Vernejoul, “Type II autosomal dominant osteopetrosis (Albers-Schonberg disease): clinical and radiological manifestations in 42 patients," Bone, vol. 26, no. 1, pp. 87-93, 2000.

[35] T. Schinke, A. F. Schilling, A. Baranowsky et al., "Impaired gastric acidification negatively affects calcium homeostasis and bone mass," Nature Medicine, vol. 15, no. 6, pp. 674-681, 2009.

[36] F. Barvencik, I. Kurth, T. Koehne et al., "CLCN7 and TCIRG1 mutations differentially affect bone matrix mineralization in osteopetrotic individuals," Journal of Bone and Mineral Research, vol. 29, no. 4, pp. 982-991, 2014.

[37] C. Supanchart, L. Wartosch, C. Schlack et al., "ClC-7 expression levels critically regulate bone turnover, but not gastric acid secretion," Bone, vol. 58, pp. 92-102, 2014.

[38] P. V. Hauschka, J. B. Lian, D. E. Cole, and C. M. Gundberg, "Osteocalcin and matrix Gla protein: vitamin K-dependent proteins in bone," Physiological Reviews, vol. 69, no. 3, pp. 9901047, 1989.

[39] J. A. Engelke, J. E. Hale, J. W. Suttie, and P. A. Price, "Vitamin K-dependent carboxylase: utilization of decarboxylated bone Gla protein and matrix Gla protein as substrates," Biochimica et Biophysica Acta, vol. 1078, no. 1, pp. 31-34, 1991.

[40] M. Ferron and J. Lacombe, "Regulation of energy metabolism by the skeleton: osteocalcin and beyond," Archives of Biochemistry and Biophysics, vol. 561, pp. 137-146, 2014.

[41] M. Ferron, J. Wei, T. Yoshizawa et al., "Insulin signaling in osteoblasts integrates bone remodeling and energy metabolism," Cell, vol. 142, no. 2, pp. 296-308, 2010.

[42] F. Oury, "A crosstalk between bone and gonads," Annals of the New York Academy of Sciences, vol. 1260, no. 1, pp. 1-7, 2012.
[43] F. Oury, G. Sumara, O. Sumara et al., "Endocrine regulation of male fertility by the skeleton," Cell, vol. 144, no. 5, pp. 796-809, 2011.

[44] P. E. Cohen, M. P. Hardy, and J. W. Pollard, "Colony-stimulating factor-1 plays a major role in the development of reproductive function in male mice," Molecular Endocrinology, vol. 11, no. 11, pp. 1636-1650, 1997.

[45] U. Lange, J. Teichmann, G. Schett, E. Neumann, and U. Müller-Ladner, "Osteoimmunology: how inflammation influences bone metabolism," Deutsche Medizinische Wochenschrift, vol. 138, no. 37, pp. 1845-1849, 2013.

[46] C. Blin-Wakkach, A. Wakkach, P. M. Sexton, N. Rochet, and G. F. Carle, "Hematological defects in the oc/oc mouse, a model of infantile malignant osteopetrosis," Leukemia, vol. 18, no. 9, pp. 1505-1511, 2004.

[47] Y.-Y. Kong, H. Yoshida, I. Sarosi et al., "OPGL is a key regulator of osteoclastogenesis, lymphocyte development and lymphnode organogenesis," Nature, vol. 397, no. 6717, pp. 315-323, 1999.

[48] N. lo Iacono, A. Pangrazio, M. Abinun et al., "RANKL cytokine: from pioneer of the osteoimmunology era to cure for a rare disease," Clinical and Developmental Immunology, vol. 2013, Article ID 412768, 9 pages, 2013.

[49] M. M. Zaiss, K. Sarter, A. Hess et al., "Increased bone density and resistance to ovariectomy-induced bone loss in FoxP3transgenic mice based on impaired osteoclast differentiation," Arthritis and Rheumatism, vol. 62, no. 8, pp. 2328-2338, 2010.

[50] Z. S. Buchwald, J. R. Kiesel, R. DiPaolo, M. S. Pagadala, and R. Aurora, "Osteoclast activated FoxP3 ${ }^{+} \mathrm{CD}^{+}$T-cells suppress bone resorption in vitro," PLoS ONE, vol. 7, no. 6, Article ID e38199, 2012.

[51] A. Del Fattore, M. Capannolo, and N. Rucci, "Bone and bone marrow: the same organ," Archives of Biochemistry and Biophysics, vol. 503, no. 1, pp. 28-34, 2010.

[52] A. Mansour, G. Abou-Ezzi, E. Sitnicka, S. E. W. Jacobsen, A. Wakkach, and C. Blin-Wakkach, "Osteoclasts promote the formation of hematopoietic stem cell niches in the bone marrow," The Journal of Experimental Medicine, vol. 209, no. 3, pp. 537549, 2012.

[53] C. Blin-Wakkach, M. Rouleau, and A. Wakkach, "Roles of osteoclasts in the control of medullary hematopoietic niches," Archives of Biochemistry and Biophysics, vol. 561, pp. 29-37, 2014.

[54] B. Lecka-Czernik and L. A. Stechschulte, "Bone and fat: a relationship of different shades," Archives of Biochemistry and Biophysics, vol. 561, pp. 124-129, 2014.

[55] S. A. Kliewer, B. M. Forman, B. Blumberg et al., "Differential expression and activation of a family of murine peroxisome proliferator-activated receptors," Proceedings of the National Academy of Sciences of the United States of America, vol. 91, no. 15, pp. 7355-7359, 1994.

[56] T. Akune, S. Ohba, S. Kamekura et al., "PPAR $\gamma$ insufficiency enhances osteogenesis through osteoblast formation from bone marrow progenitors," Journal of Clinical Investigation, vol. 113, no. 6, pp. 846-855, 2004.

[57] E. D. Rosen, P. Sarraf, A. E. Troy et al., "PPAR $\gamma$ is required for the differentiation of adipose tissue in vivo and in vitro," Molecular Cell, vol. 4, no. 4, pp. 611-617, 1999.

[58] Y. Wan, L.-W. Chong, and R. M. Evans, "PPAR- $\gamma$ regulates osteoclastogenesis in mice," Nature Medicine, vol. 13, no. 12, pp. 1496-1503, 2007. 
[59] T.-A. Cock, J. Back, F. Elefteriou et al., "Enhanced bone formation in lipodystrophic PPAR $\gamma^{\text {hyp/hyp }}$ mice relocates haematopoiesis to the spleen," EMBO Reports, vol. 5, no. 10, pp. 1007-1012, 2004.

[60] A. Neve, A. Corrado, and F. P. Cantatore, "Osteocalcin: skeletal and extra-skeletal effects," Journal of Cellular Physiology, vol. 228, no. 6, pp. 1149-1153, 2013. 


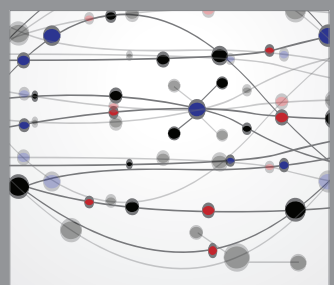

The Scientific World Journal
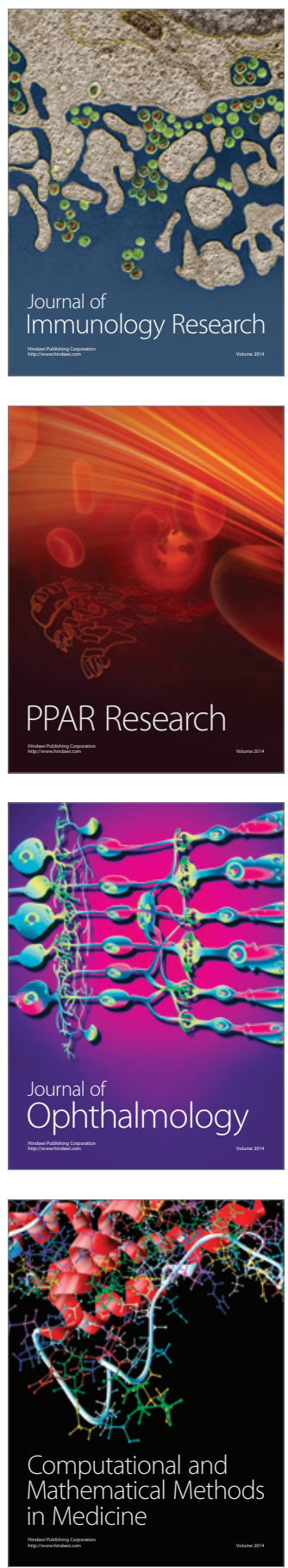

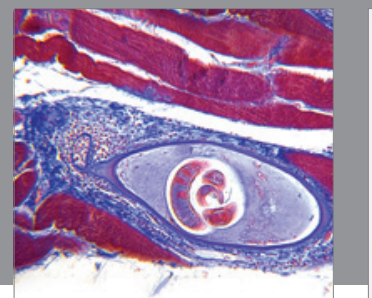

Gastroenterology

Research and Practice
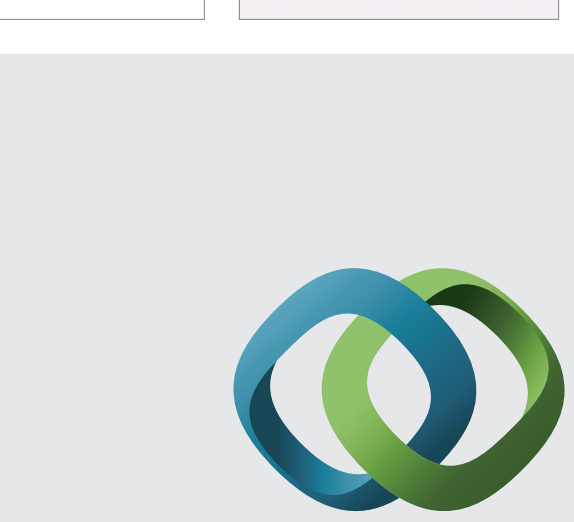

\section{Hindawi}

Submit your manuscripts at

http://www.hindawi.com
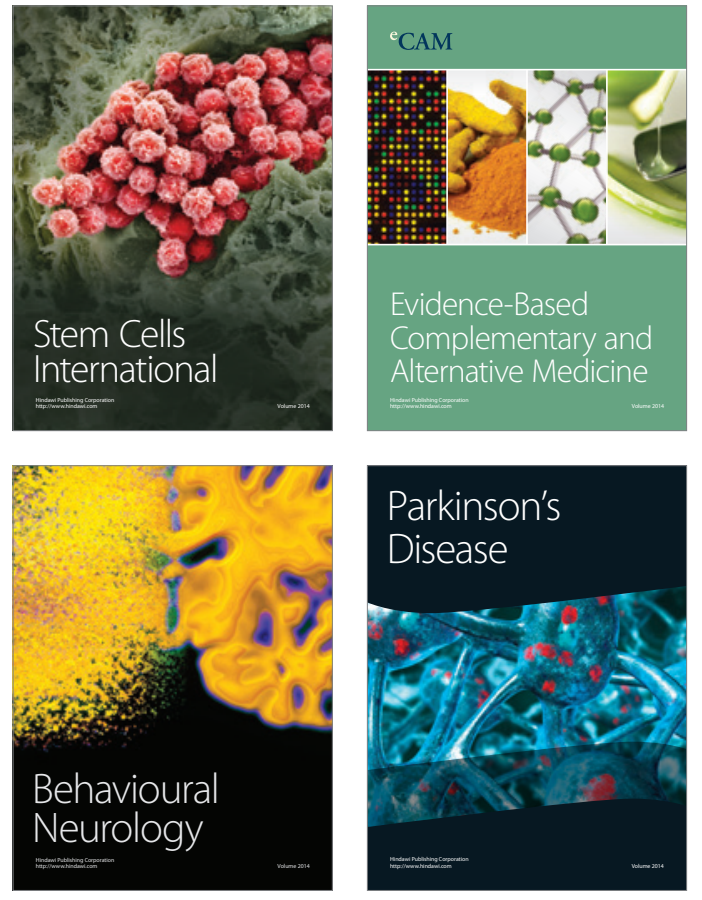
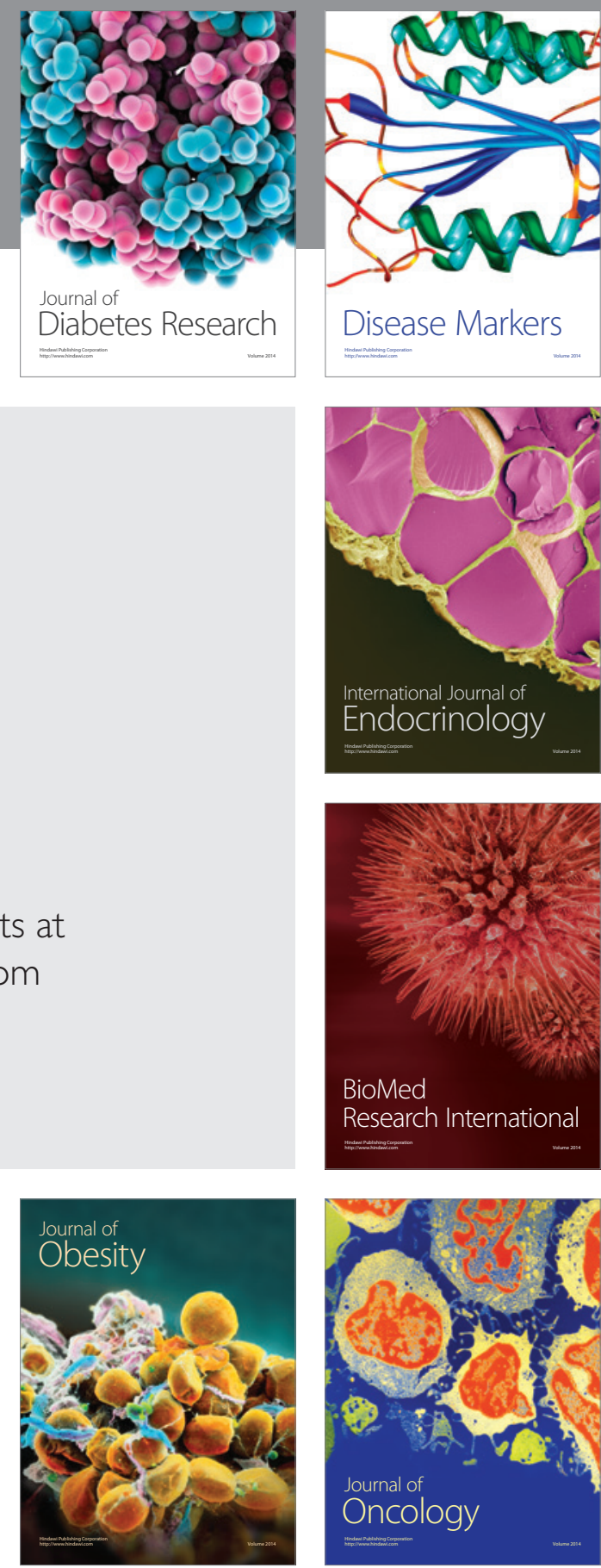

Disease Markers
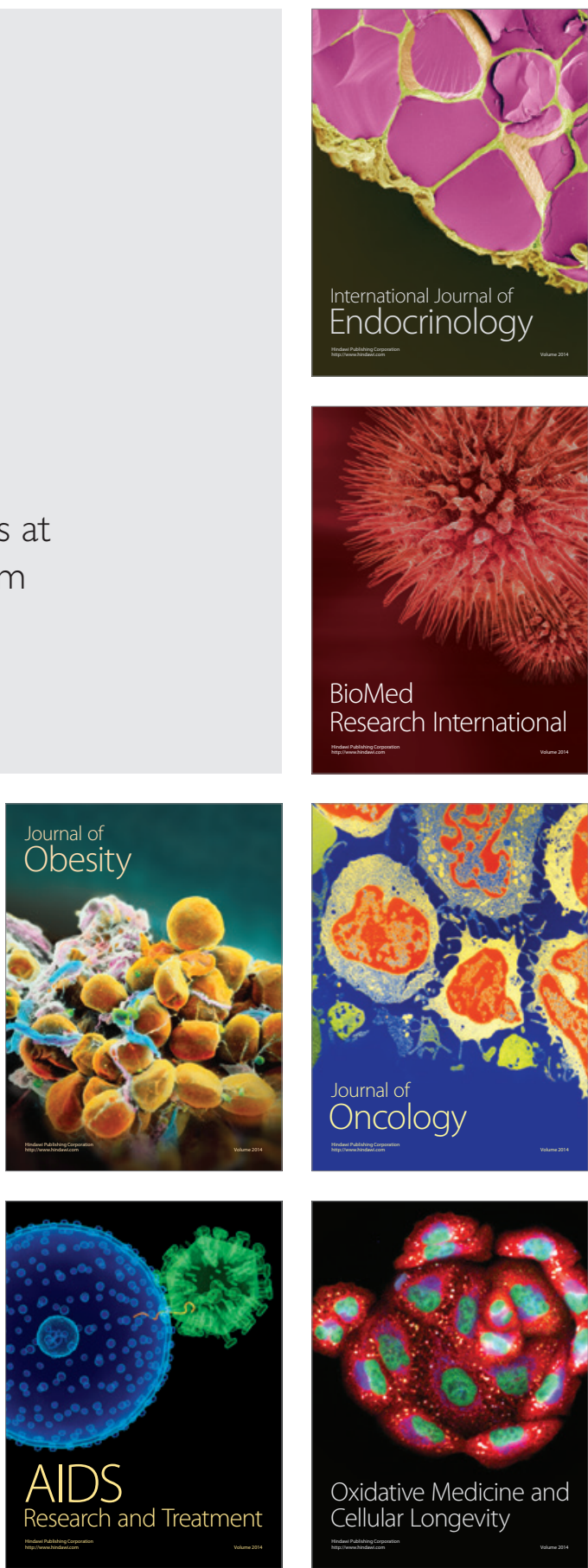